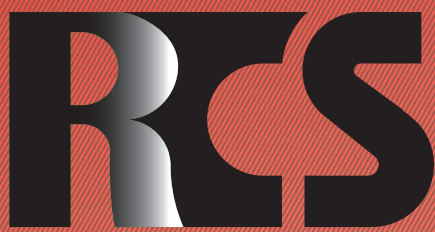

Depósito legal ppi $201502 Z U 4662$

Esta publicación científica en formato digital es continuidad de la revista impresa Depósito Legal: pp $197402 Z$ U789

- ISSN: 1315-9518 • ISSN-E: 2477-9431

Revista de Ciencias Sociales

Universidad del Zulia. Revista de la Facultad de Ciencias Económicas y Sociales Vol. XXVII,

No. 4,2021

Esta publicación científica en formato digital es continuidad de la revista impresa Depósito Legal: pp $197402 Z U 789$ ISSN: $1315-9518$ 


\title{
Gasto público y crecimiento económico en Venezuela: Un análisis de cointegración y causalidad $(1950-2017)$
}

\author{
Peña, Carlos*
}

\section{Resumen}

El gasto público es un tema importante en el desarrollo económico de un país. La significancia de este gasto en el producto ha sido ampliamente analizada en la literatura económica. En Venezuela para el lapso en estudio y, particularmente a partir de 1974, el gasto público, tomó relevancia en la economía, teniendo un efecto importante sobre el producto a largo plazo. El objetivo de esta investigación es establecer la dinámica del gasto público y el crecimiento económico, tanto en el corto como en el largo plazo en Venezuela para el lapso 1950-2017. La metodología se basó en los modelos de vectores autorregresivos cointegrados y modelos de vectores de corrección de errores. Se verificó, la existencia del enfoque keynesiano para el caso de estudio establecido. Este hallazgo es importante puesto que, puede permitir y, hasta validar el uso del gasto público como instrumento de política fiscal, lo que pudiera favorecer, vía demanda agregada, el crecimiento económico.

Palabras clave: Gasto público; causalidad; modelos de corrección de errores; enfoque keynesiano; Ley de Warner.

\footnotetext{
Doctorado y Postdoctorado en Ciencias Sociales. Economista. Profesor Titular y Director del Instituto de Investigaciones Económicas y Sociales "Dr. Rodolfo Quintero" en la Universidad Central de Venezuela (UCV), Venezuela. Profesor del Doctorado en Ciencias Económicas en la Universidad Católica Andrés Bello (UCAB), Venezuela. Investigador adscrito al Centro de Estudios de Economía Latinoamericana (CEdEL), Universidad de la Laguna, Tenerife, España. E-mail: cpenaparra@gmail. com; carlos.pena@ucv.ve ORCID: https://orcid.org/0000-0002-6187-2916
} 


\title{
Public spending and economic growth in Venezuela: An analysis of cointegration and causality $(1950-2017)$
}

\begin{abstract}
Public spending is an important issue in a country's economic development. The significance of this expenditure on output has been extensively analyzed in the economic literature. In Venezuela for the period under study and, particularly from 1974, public spending, became relevant in the economy, having an important effect on output in the long term. The objective of this research is to establish the dynamics of public spending and economic growth, both in the short and long term in Venezuela for the period 19502017. The methodology was based on co-integrated autoregressive vector models and error correction vector models. The existence of the Keynesian approach to the established case study was verified. This finding is important since it can allow and even validate the use of public spending as an instrument of fiscal policy, which could favor, via aggregate demand, economic growth.
\end{abstract}

Keywords: Public spending; causation; models of error correction; Keynesian approach; Warner's law.

\section{Introducción}

Una de las relaciones más tratadas y discutidas en los temas de crecimiento económico y desarrollo, es la relación entre el gasto público y el crecimiento económico, no solo por su importancia sino también por las controversias que existen. En este sentido, la presencia de dos tesis: La keynesiana y la wagneriana, conocida como la Ley de Warner, presenta la principal controversia que surge de la dirección de causalidad entre las variables. Keynes, plantea que el gasto público tiene un efecto positivo en la demanda efectiva $y$, por ende, sobre el crecimiento económico. Por el contrario, la Ley de Warner, sostiene que el gasto público como \% del Producto Interno Bruto (PIB) es elástico a éste y tiende a aumentar al incrementarse el crecimiento económico. En la primera, la causalidad va del gasto público al crecimiento económico; en la segunda, hay una relación del PIB al gasto público.

Diversos son los estudios que se han desarrollado en torno a estas hipótesis. La evidencia empírica muestra hallazgos a favor de ambas; sin embargo, dependerá del enfoque econométrico adoptado y del período de estudio, la validación de una u otra o de ambas. Para Venezuela, en el lapso de estudio 1950 2017, el Estado ha tenido un peso significativo en la economía del país, básicamente a partir de 1974, cuando los precios del petróleo aumentaron de manera importante. A partir de allí, la influencia del sector público ha sido preponderante; no obstante, esa relación se ha visto condicionada por la inestabilidad y volatilidad del mercado petrolero. La evidencia empírica preliminar valida una relación que va del gasto público al PIB.

En este contexto, el objetivo del trabajo consiste en establecer la dinámica entre el gasto público y el crecimiento económico, PIB, a través de los test de cointegración y causalidad, para Venezuela en el lapso de 1950 a 2017. En función de esto, la metodología está justificada en los modelos de vectores autorregresivo (VAR) cointegrados, el modelo de corrección de errores (VECM) y los test de causalidad de largo y corto plazo de Granger. La idea es verificar si el enfoque keynesiano o el de Warner se cumplen. Esto, sin entrar en 
mayores consideraciones.

El documento está organizado de la siguiente manera: Una primera parte, relacionada a los aspectos teóricos de la relación entre gasto público y crecimiento económico; una segunda, dedicada a los aspectos empíricos y metodológicos de dicha relación; una tercera, donde muestra las estimaciones, resultados, así como las dinámicas de largo y corto plazo y, finalmente, las conclusiones.

\section{Gasto público y crecimiento económico}

\subsection{Aspectos teóricos}

Uno de los primeros estudios en este campo fue el de Aschauer (1989). Este autor analizó el vínculo entre la productividad agregada y el stock y el flujo de las variables que conforman el gasto de gobierno, introduciendo para ello, el stock de capital público en la función de producción. Dado esto, concluye que el stock de infraestructura tiene un impacto sobre la productividad total de los factores, subrayando la importancia del gasto público en el crecimiento económico. A partir de allí, en los tópicos de crecimiento y desarrollo económico, el efecto del gasto público en estas variables, es uno de los más debatidos, generándose una amplia literatura, tanto teórica como empírica. En este sentido, Barro (1990), incorpora en el modelo de crecimiento endógeno, el gasto público e impuestos. Este modelo permite analizar el tamaño óptimo del gobierno, así como la relación que guarda éste con el crecimiento y la tasa de ahorro.

En la literatura especializada, dos enfoques macroeconómicos tienen particular relevancia: Uno que sostiene que el gasto público auspicia el crecimiento económico $\mathrm{y}$, el otro que plantea que el crecimiento económico es el que favorece la expansión del gasto público. Estas posturas se pueden asociar a Keynes $(1926 ; 1971)$ y a Warner
(1958), respectivamente ${ }^{(1)}$. Así, Keynes y los keynesianos $^{(2)}$ señalan que el gasto público ejerce un efecto positivo en la demanda efectiva o agregada $\mathrm{y}$, en consecuencia, sobre el PIB; así mismo, plantean que el gasto público puede ser utilizado como un instrumento contracíclico ${ }^{(3)}$ para reducir la brecha de la producción real. Como resultado, este enfoque sugiere que el gasto público tiene impactos positivos y altamente significativos en el PIB y, por supuesto, sobre el crecimiento económico.

El segundo enfoque es conocido como la Ley de Wagner, que ha despertado un gran interés entre los economistas del sector público desde su redescubrimiento a través de la recopilación realizada por Musgrave y Peacock (1958) titulada Classics in Public Finance, no obstante, Warner consideró que el aumento del gasto público proseguía una tendencia secular y es elástico con respecto al PIB. En lo que respecta a esto, varios autores han aportado en la explicación de esta idea. Entre ellos, Musgrave (1969); y Bird (1971) secundan esto, arguyendo que un grupo significativos de bienes públicos son de lujo; por lo tanto, es de suponer que el gasto sea elástico con respecto a la renta.

Por su parte, Peacock y Wiseman (1961) consiguen, para el Reino Unido, durante la primera mitad del siglo XX, que el aumento del gasto obedeció, en principio, a lo que se designó como efecto desplazamiento. Estos autores, critican a Wagner por no haber tomado en cuenta dicho resultado para justificar la expansión del gasto público. Estos dos enfoques sugieren una relación de causalidad. La hipótesis keynesiana indica que la relación va del gasto público al crecimiento económico. Por el contrario, la Ley de Wagner, señala que el lazo va desde el crecimiento hacia el gasto público, de forma tal que conforme una economía presenta un mayor crecimiento económico y logra alcanzar mayores niveles de ingreso y desarrollo, la participación del gobierno a través del gasto público dentro del PIB se incrementa. 


\subsection{Aspectos empíricos}

Los enfoques definidos en la sección anterior, dado la dirección de la causalidad, ha ocasionado que, en la literatura empírica se presenten diversas conclusiones del efecto del gasto público sobre el crecimiento económico, en términos estadísticos; sin embargo, se pueden apreciar dos metodologías en las investigaciones que han analizado las dos posturas. Según Campo y Mendoza (2018):

Las causas de estos resultados mixtos han sido atribuidas a varios aspectos, entre los que se destacan las formas funcionales de los modelos, las muestras, los métodos econométricos y los datos utilizados. Sin embargo, la razón a la que más atribuyen estos diversos resultados es a las metodologías econométricas, debido a que siempre ha existido una discusión sobre cuál de los enfoques metodológicos -corte transversal, series de tiempo o datos de panel- es el mejor para estimar las relaciones funcionales entre el gasto y el PIB. (p.80)

En la Ley de Warner y, desde el punto de vista teórico, Warner no hizo explicito la relación matemática entre ambas variables, mucho menos, una medida o definición de gasto público. En este sentido, Aparco y Flores (2019) señalan “(...) por lo que dentro de la contrastación empírica se han utilizado diversas formas funcionales y medidas del gasto público a fin de contrastar su hipótesis" (p.56).

Las investigaciones empíricas previas, se basaban en la técnica de los mínimos cuadrados ordinarios (MCO). El problema es que no se valuaba si existía una relación a largo plazo. Con el desarrollo de nuevos métodos como la cointegración (Engle y Granger, 1986; Johansen, 1988; Johansen y Joselius, 1990) y el test de causalidad de Granger (1969), se subsanaron algunos problemas que presentaban los trabajos predecesores. Los documentos de Singh y Sahni (1984); y Henrekson (1992), son considerados pioneros en incluir el contraste de cointegración y de causalidad para analizar el vínculo entre el gasto público y el PIB. Las posteriores investigaciones han adoptado este enfoque ${ }^{(4)}$.
Asimismo, las investigaciones recientes incluyen la hipótesis keynesiana, en oposición a la tesis warneriana. Entre ellos, Kaur y Afifa (2017), encuentran evidencia para la India, que muestra la presencia de ambas hipótesis. Para España, Diaz-Fuentes y Revuelta (2013), encontraron evidencia a favor de la Ley de Warner; pero plantean que:

No obstante, si acotamos el período de estudio a las 4 últimas décadas del siglo xx, es la hipótesis keynesiana la que encuentra mayor soporte empírico. El resultado, aunque pueda parecer paradójico, encuentra una explicación lógica ya que antes de la reforma fiscal de 1977, la transición a la democracia y el desarrollo del Estado de Bienestar, el peso del sector público era mucho menor, pasando, con estas transformaciones, a tener un mayor poder de influencia sobre el crecimiento económico al incrementar su tamaño relativo. (p.39)

Por otra parte, para algunos países africanos como Kenia, Ghana, Costa de Marfil, Benín, Senegal y Sudáfrica, resalta la investigación de Keho (2016), quien halla evidencia diferenciada en cuanto a la dirección de la causalidad. Para Kenia, encuentra que la relación de causalidad es bidireccional; en tanto que, Ghana y Costa de Marfil, se verifica la Ley de Warner. En los países de Sudáfrica, Benín y Senegal, muestra que la causalidad va del PIB per cápita, al gasto público; sin embargo, la elasticidad no es positiva.

Para América Latina, hay evidencia que apoya ambas tesis. Aparco y Flores (2019), validan la tesis keynesiana para el corto plazo "(...) ello es trascendente, puesto que se recomienda y valida la usanza de utilizar al gasto público como instrumento de política fiscal, lo que a su vez ayudaría a minimizar la brecha entre el PIB y el PIB potencial" (p.69). Para Bolivia, Molina y Gantier (2015), encuentran evidencia del cumplimiento de la teoría keynesiana. Para Colombia, en el lapso 1984-2012, Campo y Mendoza (2018), señalan, según los resultados, la validez de la hipótesis keynesiana para ese país. Para México, Salazar (2020), aunque no lo plantea explícitamente, verifica la hipótesis de Keynes, tanto en el corto como en el largo plazo. 
Así, de acuerdo con Salazar (2020): "El gasto público total, y por compontes entre gasto de corriente y de capital, tienen un impacto positivo y estadísticamente significativo de corto y largo plazos sobre la actividad económica en México, en el período 1995-2018" (p.68). Por su parte, Comín, Díaz y Revuelta (2009), muestran evidencia para Argentina, Brasil, España y México durante el siglo XX, que la Ley de Warner se cumplió para esos países, y comentan que: "Una explicación plausible es que cuando el tamaño del sector público es reducido, resulta difícil que pueda influir directamente en la evolución de la producción. Este ha podido ser el caso de las cuatro economías consideradas" (p.11-12).

\section{Gasto público y crecimiento económico. La evidencia empírica}

\subsection{Aspectos teóricos y metodológicos}

El lapso de estudio está comprendido entre los años 1950 y 2017. Un lapso lo suficientemente largo y particularmente diferenciado en cuanto al comportamiento macroeconómico. Un período que ha estado caracterizado por etapas de estabilidad y crecimiento sostenido; otro, de crecimiento acelerado, seguido de una etapa de desaceleración y estancamiento económico; un cuarto, de estancamiento y recesión, volatilidad e inestabilidad económica, crisis bancarias, choques externos negativos; el de 1999 a 2017, que se ha caracterizado por cambios institucionales, crisis mundial, choques externos e internos, positivos y negativos, un incremento en la intervención del Estado, principalmente por medio de una política económica discrecional y controles sobre la economía.

En este contexto, la evolución del gasto público puede tener efectos positivos en el crecimiento económico. El gasto público, entre ellos el de infraestructura, puede generar externalidades positivas sobre el crecimiento, apuntalando el crecimiento a largo plazo (Peña, 2018). Así, la hipótesis teórica que se plantea es que, el crecimiento económico, medido por el PIB per cápita (PIBpc), está afectado positivamente por el gasto público, tanto en el corto como en el largo plazo. Para aproximarse al vínculo entre el gasto público y el crecimiento, se presentan los siguientes gráficos.

En el Gráfico I, se muestra la relación entre el PIBpc y el gasto público (GP). Se observa que hay una relación positiva y significativa, para el lapso de estudio, con una correlación del 64,5\%. Más allá del análisis que se pueda hacer al interior de la relación, lo cual no está previsto, se puede plantear que hay evidencia preliminar a favor de la tesis keynesiana, cuya causalidad va del gasto público al PIBpc. En este caso, el PIBpc responde a incrementos en el GP. 


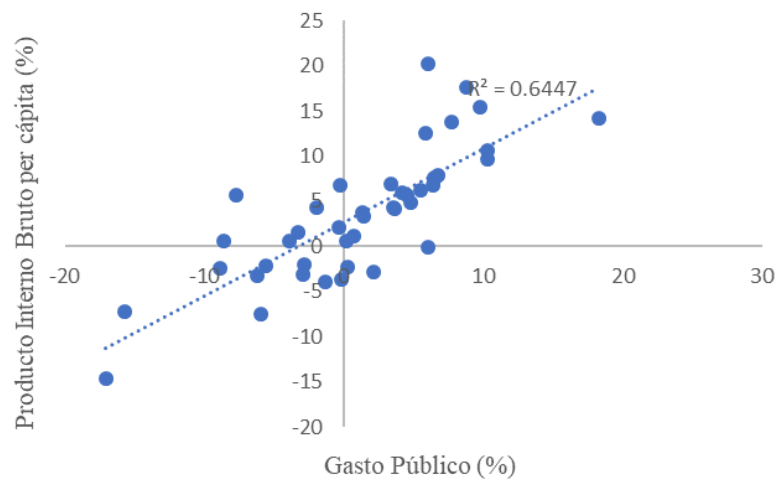

Fuente: Elaboración propia, 2020 en base a Baptista (2011); y datos del Banco Central de Venezuela.

Gráfico I: Producto Interno Bruto per cápita y gasto público. Venezuela, 1950 2017

En el siguiente Gráfico II, se presenta la relación entre el PIBpc y el gasto; es decir, lo que establece la Ley de Warner, quien plantea que la relación de causalidad va del producto al gasto. Como se observa, la relación es positiva, pero poco significativa. La relación entre ambas es de apenas $15,7 \%$. En todo caso, la evidencia parece muy débil a favor de esta hipótesis.

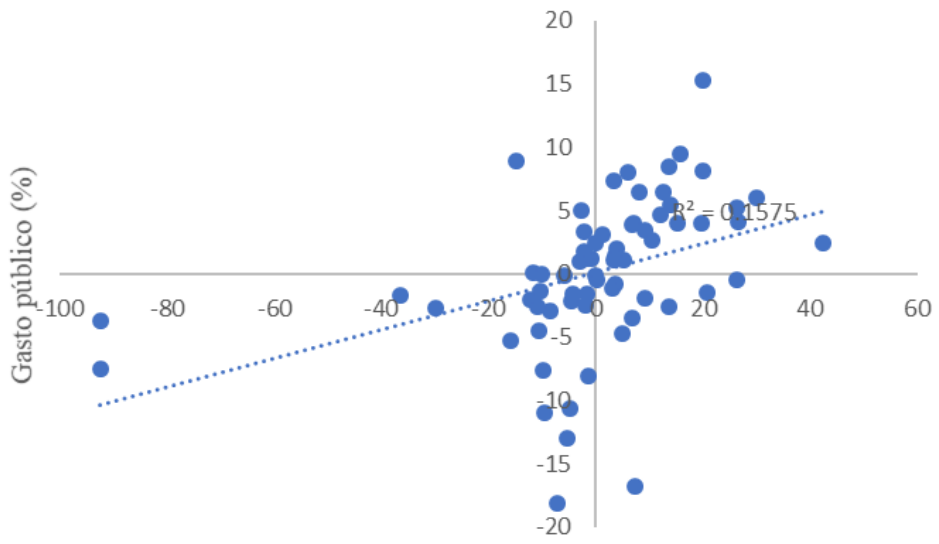

Producto Interno Bruto per cápita (\%)

Fuente: Elaboración propia, 2020 en base a Baptista (2011); y cifras del Banco Central de Venezuela. Gráfico II: Producto interno Bruto per cápita y gasto público. Venezuela, 1950 2017 
Sobre la base de las consideraciones anteriores, se hace necesario verificar, de manera más exhaustiva, estas hipótesis. Así, la estrategia econométrica está basada en las técnicas econométricas para series de tiempo. La metodología a seguir será el de la cointegración, dado el enfoque de esta investigación $\mathrm{y}$, teniendo en cuenta trabajos previos. En este sentido, se procedió a realizar pruebas concernientes con el enfoque planteado; posteriormente, se estimó un vector de corrección de errores (VECM), para determinar las relaciones de corto y largo plazo entre las variables en estudio; así mismo, se utilizó la técnica formulada por Granger (1969), para obtener las relaciones de causalidad entre el ingreso y el gasto público.

No obstante, es importante verificar si las variables son estacionarias o no; es decir, verificar el grado de integración de cada una de las series en estudio. Los resultados permiten lo siguiente: Si son integradas de orden I(0); es decir, las variables son estacionarias en niveles, se emplean las series en niveles para estimar un modelo de vectores autorregresivos (VAR). Si las variables son no estacionarias, pero integradas del mismo orden se comprueba, mediante los test de cointegración, si existe una relación de largo plazo entre las series, si lo hay, lo correcto será estimar un VECM. Si las variables no cointegran, se procede a estimar un VAR, con las variables en diferencia (Aparco y Flores, 2019). En el Cuadro 1, se muestran las variables según la versión de Ley de Warner, siguiendo a Comín et al. (2009); Diaz-Fuentes y Revuelta (2013); Aparco y Flores (2019); entre otros.

\section{Cuadro 1}

\section{Especificaciones de la Ley de Warner}

\begin{tabular}{ll}
\hline \multicolumn{1}{c}{ Versión } & \multicolumn{1}{c}{ Variables } \\
\hline Peacock y Wiseman (1961) & $\ln G=\alpha+\beta \operatorname{lnpib}$ \\
Goffman (1968) & $\ln G=\alpha+\beta \operatorname{lnpibpc}$ \\
Musgrave (1969) & $\ln G_{-}$pib $=\alpha+\beta \operatorname{lnpib}$ \\
Gupta (1967) & $\ln G_{-}$pib $=\alpha+\beta \operatorname{lnpibpc}(4)$ \\
Mann (1980) & $\ln G p c=\alpha+\beta \operatorname{lnpibpc}$ \\
\hline
\end{tabular}

Nota: $\ln \mathrm{G}=$ logaritmo de gasto público; lnpib=logaritmo del producto interno bruto; lnG_pib=logaritmo del gasto público como \% del PIB; lnpibpc= logaritmo del producto interno bruto per cápita; $\operatorname{lngpc}=\operatorname{logaritmo}$ del gasto público per cápita.

Fuente: Elaboración propia, 2020.

Se puede observar que las distintas especificaciones vinculan variables cercanas, pero no parecidas. Las ecuaciones (1) y (2), corresponden a la versión absoluta de la Ley de Warner. La (3) y la (4) a la versión relativa, y la (5) a la absoluta per cápita. En cuanto a la postura keynesiana, esta afirma que el aumento en el gasto público conlleva a un incremento en el crecimiento económico; por lo tanto, se puede notar la inversión en la dirección de la causalidad en la hipótesis de Warner y la de Keynes. De tal manera que, las funciones anteriores sirven para contrastar esta última, sustituyendo la variable dependiente por la explicativa. lpib $=f(l g)$. de acuerdo con Comín et al. (2009).

En función de lo anterior, las variables a utilizar en este trabajo serán: El gasto público ${ }^{(5)}$, definido en este trabajo como la suma del consumo final del Gobierno Central ${ }^{(6)}$ y la inversión bruta fija pública. Ambas variables están expresadas en términos reales (Aparco y Flores, 2019); así mismo, las series del Producto Interno Bruto total (PIB) y el per cápita (PIBpc).

Magazzino (2012); y Molina y Gartier (2015), plantean que se pueden 
establecer cuatro hipótesis en cuanto a la relación de causalidad entre gasto público y crecimiento económico, a saber: Hipótesis de neutralidad, la de Warner, la de Keynes y la de retroalimentación. La primera, indica que no hay relación entre las variables mencionadas; es decir, el vínculo entre ellas es nulo; la de Warner, como se mencionó en párrafos anteriores, dice que el crecimiento económico causa al gasto público; la de Keynes, sostiene que la causalidad es inversa a la de Warner; y, por último, la de retroalimentación, señala que la causalidad es bidireccional, esto es, el crecimiento económico causa el gasto público y viceversa.

\section{Estimación y resultados: dinámicas de corto y largo plazo}

\subsection{Estacionariedad e integración}

A continuación, se procede a verificar cuál es el orden de integración ${ }^{(7)}$ de las variables empleadas en el análisis. Para ello, se procede a efectuar las pruebas de raíz unitaria, las cuales se realizan para el logaritmo natural de las series señaladas en el Cuadro 1. Los test para evaluar si las variables son estacionarias, son el de Dickey-Fuller Ampliado (ADF) (Dickey y Fuller, 1979; 1981); el de correcciones no paramétricas de Phillips-Perron (PP) (Phillips y Perron, 1988). Los resultados se muestran en la Tabla 1, como se observa, la aplicación de dichas pruebas permite afirmar que las primeras diferencias de las variables analizadas son estacionarias. Es decir, todas las variables son integradas de orden uno (I(1)).

Tabla 1

Test de raíz unitaria

\begin{tabular}{ccc}
\hline \multicolumn{3}{c}{ Niveles } \\
\hline ADF & PP \\
\hline Variable & P-valor & P-valor \\
\hline
\end{tabular}

\section{Cont... Tabla 1}

\begin{tabular}{lcc}
\hline Lnpib & 0,1258 & 0,3985 \\
\hline Lnpibpc & 0,5538 & 0,4515 \\
\hline Lncpub & 0,4297 & 0,2380 \\
\hline lncpub_pib & 0,4457 & 0,1442 \\
\hline Lncpubpc & 0,8475 & 0,6815 \\
\hline & Diferencias & \\
\hline$\Delta$ lnpib & 0,0023 & 0,0005 \\
\hline$\Delta$ lnpibpc & 0,0001 & 0,0007 \\
\hline$\Delta$ lncpub & 0,0002 & 0,0000 \\
\hline$\Delta$ lncpub_pib & 0,0050 & 0,0000 \\
\hline$\Delta$ lncpubpc & 0,0002 & 0,0003 \\
\hline
\end{tabular}

Nota: Nivel de significancia estadística: 1\%; ADF: Dickey-Fuller Aumentado; PP: Phillips-Perron; Hipótesis nula: ADF; PP: la variable tiene raíz unitaria; D: primera diferencia de la variable en estudio.

Fuente: Elaboración propia, 2020 a partir de las estimaciones Eviews.

Una vez confirmada la existencia que las variables son estacionarias en primeras diferencias, se procede a verificar si las series en estudio cointegran; es decir, presentan un vínculo de largo plazo. Para ello, se procedió a estimar un VAR cointegrado y verificar los supuestos de ruido blanco sobre los residuos; así mismo, las pruebas para confirmar que dicho VAR es estable. Dichas pruebas conllevan a concluir que el VAR cumple con los criterios establecidos.

Luego, se procedió a aplicar la prueba de cointegración de Johansen (1988); y Johansen y Joselius (1990). Es importante mencionar que se incluyeron tres variables dummies ${ }^{(8)}$ en la estimación del vector de cointegración, para modelar los quiebres estructurales que presentaron las variables. Las pruebas se aplicaron para las diferentes versiones de la Ley de Warner; sin embargo, las pruebas de cointegración para estas especificaciones, no resultaron estadísticamente significativas, lo cual implica que no hay relación a largo plazo, según esta hipótesis. En consecuencia, 
Gasto público y crecimiento económico en Venezuela: Un análisis de cointegración y

causalidad (1950 - 2017)

se procedió a evaluar la hipótesis keynesiana. los resultados siguiendo las especificaciones En la Tabla 2 a continuación, se presentan disponibles en el Cuadro 1.

Tabla 2

Resultados de la prueba de cointegración de Johansen

\begin{tabular}{|c|c|c|c|c|}
\hline \multirow[b]{2}{*}{ Ecuación } & \multicolumn{4}{|c|}{ Test } \\
\hline & Hipótesis nula & Traza & Valor Critico & P-varlor** \\
\hline \multirow{5}{*}{1} & $\mathrm{r}=0$ & 14,8090 & 12,3209 & 0,0188 \\
\hline & $r \leq 0$ & 3,4379 & 4,1299 & 0,0755 \\
\hline & & Max Valor & & \\
\hline & $\mathrm{r}=0$ & 12,3691 & 11,2248 & 0,0372 \\
\hline & $r \leq 0$ & 3,4379 & 4,1299 & 0,0755 \\
\hline \multirow{6}{*}{2} & & Traza & & \\
\hline & $\mathrm{r}=0$ & 16,9544 & 15,4947 & 0,0212 \\
\hline & $r \leq 0$ & 0,6085 & 0,6085 & 0,4353 \\
\hline & & Max Valor & & \\
\hline & $\mathrm{r}=0$ & 15,3559 & 14,2646 & 0,0300 \\
\hline & $r \leq 0$ & 0,6085 & 3,8415 & 0,4353 \\
\hline & & Traza & & \\
\hline & $\mathrm{r}=0$ & 15,4947 & 12,0942 & 0,0153 \\
\hline & $\mathrm{r} \leq 1$ & 0,2641 & 3,8415 & 0,6073 \\
\hline \multirow{3}{*}{3} & & Max valor & & \\
\hline & $\mathrm{r}=0$ & 14,2646 & 13,2646 & 0,0172 \\
\hline & $\mathrm{r} \leq 1$ & 0,2641 & 3,8415 & 0,6073 \\
\hline \multirow{5}{*}{4} & & Traza & & \\
\hline & $\mathrm{r}=0$ & 17,7361 & 15,4947 & 0,0218 \\
\hline & $\mathrm{r} \leq 1$ & 2,9864 & 3,8415 & 0,0840 \\
\hline & $=0$ & Max valor & 140 & ת \\
\hline & 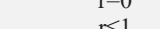 & 15,4738 & 14,2646 & 0,0320 \\
\hline \multirow{5}{*}{5} & & $\begin{array}{c}2,9804 \\
\text { Traza }\end{array}$ & 3,8413 & 0,0840 \\
\hline & $\mathrm{r}=0$ & 17,3231 & 15,4947 & 0,0245 \\
\hline & $r \leq 1$ & 2,8841 & 3,8415 & 0,0895 \\
\hline & $r=0$ & $\begin{array}{c}\text { Max Valor } \\
16,4390\end{array}$ & 14,2646 & 0,0335 \\
\hline & $<<1$ & 28841 & 38816 & 00895 \\
\hline
\end{tabular}

Nota: *0,05,**MacKinnon-Haug-Michelis (1999) P-values

Fuente: Elaboración propia, 2020, a partir de las estimaciones Eviews.

Como se aprecia en la Tabla 2, la prueba de la traza rechaza, con un nivel de significancia de $5 \%$ la existencia de cero ecuaciones de cointegración. Igualmente, no fue posible rechazar la hipótesis nula que imponen máximo 1 relaciones de cointegración. En consecuencia, esta prueba indicó que existe una ecuación de cointegración para las variables en estudio. La prueba del máximo valor propio permitió concluir lo mismo. Como resultado, se concluye que existe relación de cointegración para cada una de las versiones.

De acuerdo con la prueba de Johansen, el rango de cointegración de las variables analizadas es $\mathrm{r}=1$ bajo la especificación de intercepto en la ecuación de cointegración, tendencia lineal en los datos y dos rezagos en los términos VAR. Con esta información se estimó el modelo de corrección de errores, VECM cointegrado.

\subsection{Dinámica de largo y corto plazo}

El hecho de que se haya verificado que dichas variables presentan el mismo orden de integración y que existe una ecuación de cointegración entre ellas, permite concluir que dicha relación de largo plazo existe 
y que es posible estimar un modelo de corrección de errores (VECM), que describa el comportamiento de las variables a lo largo del tiempo. Se estimó un VECM $(1,2)$. Esto, permitió que los resultados obtenidos en el VECM, fueran estadísticamente satisfactorios. Esto implicó que, se acepta la hipótesis nula de normalidad, no autocorrelación y homocedasticidad en los residuos; además, el VECM presenta estabilidad en sus parámetros. Desde el punto de vista económico, también resultó satisfactorio. En la Tabla 3, se muestran los resultados.

Tabla 3

Estimación del vector de corrección de errores

\begin{tabular}{|c|c|c|c|c|c|c|c|c|c|c|}
\hline & \multicolumn{10}{|c|}{ Modelos } \\
\hline & & 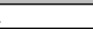 & & & & & & 4 & & 5 \\
\hline $\begin{array}{ll}\begin{array}{l}\text { Variables del } \\
\text { sistema }\end{array} & \\
\end{array}$ & lpib & lcpub & lpibpc & lcpub & lpib & $\begin{array}{l}\text { lcpub/ } \\
\text { pib }\end{array}$ & lpibpc & $\begin{array}{l}\text { lcpub/ } \\
\text { pib }\end{array}$ & Lpibpc & lcpupc \\
\hline $\begin{array}{l}\text { Componentes } \\
\text { del vector de } \\
\text { cointegración } \\
\text { estimado } \beta\end{array}$ & 1 & $\begin{array}{c}-0,2823 \\
(0,1155 \\
{[2,4446]}\end{array}$ & 1 & $\begin{array}{l}-0,0286 \\
(0,0240) \\
{[1,1917]}\end{array}$ & 1 & $\begin{array}{c}-0,3853 \\
(0,1692) \\
{[2,2692]}\end{array}$ & 1 & $\begin{array}{l}-0,0997 \\
(0,0467) \\
{[2,1340]}\end{array}$ & 1 & $\begin{array}{l}-0,0875 \\
(0,0452) \\
{[2,1075]}\end{array}$ \\
\hline $\begin{array}{l}\text { Componentes } \\
\text { del vector de } \\
\text { velocidad de } \\
\text { ajustes } \alpha\end{array}$ & $\begin{array}{r}-0,0461 \\
(0,0123) \\
{[-3,7385]}\end{array}$ & $\begin{array}{r}-0,1350 \\
(0,1490) \\
{[-0,9066]}\end{array}$ & $\begin{array}{r}-0,2391 \\
(0,0736) \\
{[-3,2498]}\end{array}$ & $\begin{array}{r}-0,7394 \\
(0,8254) \\
{[-0,8958]}\end{array}$ & $\begin{array}{r}-0,0323 \\
(0,0089) \\
{[-3,7385]}\end{array}$ & $\begin{array}{r}-0,0642 \\
(0,1062) \\
{[-0,6051]}\end{array}$ & $\begin{array}{r}-0,1951 \\
(0,0555) \\
{[-3,5119]}\end{array}$ & $\begin{array}{c}-0,2720 \\
(0,6233) \\
{[-0,4364]}\end{array}$ & $\begin{array}{r}-0,2078 \\
(0,0591) \\
{[-3,5133]}\end{array}$ & $\begin{array}{r}-0,4887 \\
(0,6700) \\
{[-0,7287]}\end{array}$ \\
\hline
\end{tabular}

Fuente: Elaboración propia, 2020 en base a las estimaciones Eviews.

Como se observa en la Tabla 3, la relación de largo plazo entre el logaritmo del lpib, lpib y de las variables cpub, lcpub, es inelástica y estadísticamente significativo, tanto al $1 \%$ como al $5 \%$ en las cinco versiones. El vector estimado resulta ser:

$$
\widehat{\beta^{\prime}}=(1,000-0,2823)
$$

Dicho vector se ha normalizado para que el primer coeficiente sea igual a 1. Con el ordenamiento del vector de variables cointegradas, se tiene que el equilibrio de largo plazo del sistema puede representarse como sigue:

$$
\text { lpib }_{t}-0,2823 \text { lcpub }_{t}=0
$$

Esto es, la combinación lineal es una serie estacionaria. Ahora bien, esta se puede reescribir como:

$$
\text { lpib }_{t}=0,2823 \text { lcpub } b_{t}
$$

La expresión describe la relación económica de largo plazo. De modo que, existe una relación estable a largo plazo entre el logaritmo del producto interno bruto y el logaritmo del gasto público. Este mismo procedimiento se sigue para las otras versiones. Quedando la relación de largo plazo como sigue:

$$
\begin{aligned}
& \text { lpibpc }_{t}=0,0286 l c p u b_{t} \\
& \text { lpib }_{t}=0,3853 \text { lcpub_pib } \\
& \text { lpibpc }_{t}=0,0997 l c p u \_p i b_{t} \\
& \text { lpibpc }_{t}=0,0875 \text { lcpubpc }
\end{aligned}
$$

Los resultados anteriores validan la existencia de un nexo positivo entre estas variables. Es evidente que a largo plazo un aumento del gasto público, en sus diversas especificaciones, generarían incremento en el PIB, siendo la versión tres (3) la que más peso tiene.

Con respecto al vector $\alpha$ estimado de velocidades de ajuste, que se muestra en la Tabla 3, los coeficientes de la velocidad de ajuste de las series lcpub, lcpub_pib y lсpupc resultaron no significativos, lo que implica que 
estas ecuaciones no aportan a la restauración del equilibrio. Por su parte, los coeficientes del lpib y lpibpc, resultaron con el signo correcto y estadísticamente significativo al 5\%, lo cual refuerza la hipótesis de la existencia de una relación de largo plazo entre las variables del modelo. Así mismo, los coeficientes del VECM, tanto del lpib como del lpibpc, presentan diversos valores, dadas las especificaciones tomadas. Esto implica, disímiles velocidades de ajuste a largo plazo.

En todo caso, los bajos coeficientes lo que denotan es que el ajuste a largo plazo de las variables es lento. Las condiciones macroeconómicas desfavorables, vigentes en el lapso de estudio, condicionan el resultado.
Los desequilibrios fiscales, monetarios, cambiarios, choques externos e internos, tienen efectos importantes en el ajuste a largo plazo.

Por su parte, la dinámica de corto plazo se muestra en la Tabla 4. Allí se evidencia que, la interacción de corto plazo está marcada por los valores rezagados del lpib y el lpibpc y el gasto público, lсpub en sus diferentes versiones. La evidencia señala que existe una relación positiva y estadísticamente significativa, entre las variables. No obstante, se observa que el impacto del gasto público es muy bajo; así mismo, se muestra que los valores rezagados del lpib y lpibpc, tienen un mayor impacto en el corto plazo.

\section{Tabla 4}

Dinámica de corto plazo

\begin{tabular}{|c|c|c|c|c|c|c|c|}
\hline \multirow[b]{2}{*}{ Modelo } & & \multicolumn{6}{|c|}{ Coeficientes } \\
\hline & & MCE & Dlpib $_{t-1}$ & Dlpibpc $_{t-1}$ & Dlcpub $_{t-1}$ & Dlcpub/pib $_{t-1}$ & Dlepubpc $_{t-1}$ \\
\hline 1 & Dlpib & $\begin{array}{c}-0,0461 \\
(0,0123) \\
{[-3,7385]}\end{array}$ & $\begin{array}{c}0,5054 \\
(0,1290) \\
{[3,9172]}\end{array}$ & & $\begin{array}{c}0,0164 \\
(0,0080) \\
{[2,0721]}\end{array}$ & & \\
\hline 2 & Dlpibpc & $\begin{array}{r}-0,2391 \\
(0,0736) \\
{[-3,2498]}\end{array}$ & & $\begin{array}{c}0,4647 \\
(0,1304) \\
{[3,5605]}\end{array}$ & $\begin{array}{c}0,0412 \\
(0,5078) \\
{[2,0501]}\end{array}$ & & \\
\hline 3 & Dlpib & $\begin{array}{c}-0,0323 \\
(0,0089) \\
{[-3,7385]}\end{array}$ & $\begin{array}{l}0,3752 \\
(0,1288) \\
{[2,9142]}\end{array}$ & & & $\begin{array}{c}0,0934 \\
(0,0379) \\
{[2,5110]}\end{array}$ & \\
\hline 4 & Dlpibpc & $\begin{array}{c}-0,1951 \\
(0,0555) \\
{[-3,5119]}\end{array}$ & & $\begin{array}{l}0,4102 \\
(0,1231) \\
{[3,3327]}\end{array}$ & & $\begin{array}{c}0,0752 \\
(0,0531) \\
{[2,1422]}\end{array}$ & \\
\hline 5 & Dlpibpc & $\begin{array}{c}-0,2078 \\
(0,0591) \\
{[-3,5133]}\end{array}$ & & $\begin{array}{l}0,4248 \\
(01246) \\
{[3,4087]}\end{array}$ & & & $\begin{array}{c}0,0698 \\
(0,0332) \\
{[2,1076]}\end{array}$ \\
\hline
\end{tabular}

Fuente: Elaboración propia, 2020 en base a las estimaciones Eviews.

\subsection{Causalidad de largo y corto plazo}

Granger (1969) $)^{(9)}$, plantea que, para verificar la relación causal entre dos variables, se debe recurrir a la estimación del VECM. Se dice entonces que, existe una relación de causalidad en el largo plazo, cuando el parámetro del VECM es negativo y estadísticamente significativo; por su parte, la causalidad de corto plazo se determinaría por la significancia conjunta de los coeficientes de las variables independientes, rezagadas y diferenciadas.

Vera y Kristjanpoller (2017), exponen que, las pruebas de causalidad de Granger de corto plazo se ejecutan a través del test de Wald para los parámetros que acompañan a las primeras diferencias rezagadas de las variables independientes; mientras que, para la causalidad de largo plazo, se realiza mediante una prueba $\mathrm{t}$ al parámetro que acompaña al término corrector de error. En las tablas 
siguientes, se muestra la evidencia de lo anterior.

En la Tabla 5, se muestran los resultados de causalidad en el largo plazo. Se exhiben los coeficientes estimados del VECM y su respectivo estadístico $t$, en las diferentes versiones. Se observa que, los coeficientes del VECM, son todos negativos; sin embargo, solo son estadísticamente significativo, al 5\%. Así, la evidencia señala que existe una relación unidireccional de causalidad, la cual va del gasto público lcpub, al logaritmo del producto interno bruto lpib, en las cinco versiones; por lo tanto, se puede concluir que existe suficiente evidencia estadística para afirmar que en el largo plazo la variable lcpub causa al lpib y el lpibpc en sentido Granger. En este caso, se puede afirmar que la Ley de Warner no se cumple para el lapso de estudio, ratificando la hipótesis keynesiana.

Tabla 5

\section{Causalidad de largo plazo}

\begin{tabular}{clcc}
\hline Versión & Dirección de causalidad & Coeficiente del MCE & t-estadístico \\
\hline \multirow{2}{*}{1} & lpib $\rightarrow$ lcpub & $-0,1350$ & $-0,9066$ \\
& lcpub $\rightarrow$ lpib & $-0,0461$ & $-3,7385^{* *}$ \\
\multirow{2}{*}{2} & lpibpc $\rightarrow$ lcpub & $-0,7394$ & $-0,8958$ \\
& lcpub $\rightarrow$ lpibpc & $-0,0391$ & $-3,2498^{* *}$ \\
\multirow{2}{*}{3} & lpib $\rightarrow$ lcpub_pib & $-0,0642$ & $-0,6051$ \\
& lcpub_pib $\rightarrow$ lpib & $-0,0324$ & $-3,7385^{* *}$ \\
\multirow{2}{*}{4} & lpibpc $\rightarrow$ lcpub_pib & $-0,2720$ & $-0,4364$ \\
& lcpub_pib $\rightarrow$ lpibpc & $-0,0351$ & $-3,3519^{* *}$ \\
\multirow{2}{*}{5} & lpibpc $\rightarrow$ lcpubpc & $-0,4887$ & $-0,7287$ \\
& lcpubpc $\rightarrow$ lpibpc & $-0,0378$ & $-3,5133^{* *}$ \\
\hline
\end{tabular}

Nota: Nivel de significancia, ${ }^{* *}$ al 5\%. El símbolo $\rightarrow$ denota el sentido de causalidad entre las variables en niveles.

Fuente: Elaboración propia, 2020 en base a estimaciones de Eviews.

En la Tabla 6, se presenta la evidencia de la relación de causalidad corto plazo. Esta, parece señalar que existe relación de causalidad solo en la versión 1 y 3 . Es decir, la causalidad va de la tasa de crecimiento del gasto público (Dlcpub), a la tasa de crecimiento del PIB (Dlpib). En el otro caso, va de la tasa de variación del gasto público como \% del PIB (Dlcpub/pib), a la tasa de variación del PIB (Dlpib). 


\begin{tabular}{cllc}
\multicolumn{5}{c}{ Tabla 6 } \\
\multicolumn{5}{c}{ Causalidad de corto plazo } \\
\hline \multirow{2}{*}{ Versión } & \multicolumn{1}{c}{$\begin{array}{c}\text { Dirección de } \\
\text { causalidad }\end{array}$} & Chi-sq & Prob \\
\hline \multirow{2}{*}{1} & Dlcpub $\rightarrow$ Dlpib & $5,1872^{* * *}$ & $0,0450^{* *}$ \\
& Dlpib $\rightarrow$ Dlcpub & 0,9860 & 0,6108 \\
\multirow{2}{*}{2} & Dlcpub $\rightarrow$ Dlpibpc & 2,6798 & 0,2619 \\
& Dlpibpc $\rightarrow$ Dlcpub & 0,6719 & 0,7146 \\
3 & Dlcpub/pib $\rightarrow$ Dlpib & $5,1842^{* * *}$ & $0,0350^{* *}$ \\
& Dlpib $\rightarrow$ Dlcpub/pib & 1,3460 & 0,5103 \\
4 & Dlcpub/pib $\rightarrow$ Dlpibpc & 3,4321 & 0,1798 \\
& Dlpibpc $\rightarrow$ Dlcpub/pib & 1,0767 & 0,5837 \\
5 & Dlcpubpc $\rightarrow$ Dlpibpc & 3,2929 & 0,1927 \\
& Dlpibpc $\rightarrow$ Dlcpubpc & 0,7491 & 0,6876 \\
\hline
\end{tabular}

Nota: Nivel de significancia, $* *$ al 5\%; *** al 10\%; El símbolo $\rightarrow$ denota el sentido de causalidad entre las variables.

Fuente: Elaboración propia, 2020 en base a las estimaciones Eviews.

\section{Conclusiones}

El objetivo de la investigación se centró en establecer la relación entre el gasto público y el crecimiento económico, a través de un análisis de cointegración y causalidad, empleando datos de series de tiempo de Venezuela, para el lapso 1950 2017. En este sentido, se tienen dos enfoques macroeconómicos: La Ley de Warner y la hipótesis keynesiana. Ambas concuerdan en que prevalece una correlación importante entre el PIB y el gasto público; no obstante, hay una diferencia significativa, la cual se expresa en el sentido de la causalidad. Así, la tesis keynesiana asume que la relación es del gasto público al PIB, lo contrario aplica para Ley de Warner.

Para Venezuela, en el lapso estudiado, la evidencia sugiere que, prevalece el enfoque keynesiano, tanto a corto como a largo plazo. Esto se verificó mediante los test de cointegración y de causalidad de Granger, para las cinco especificaciones de la Ley de Warner.

De las cinco especificaciones, la tercera, la cual se expresa con el gasto como \% del PIB, mostró un mayor impacto sobre el PIB a largo plazo. En cuanto a la relación de corto plazo, el efecto del gasto público sobre el PIB y PIBpc, es muy bajo, pero estadísticamente significativos. En cuanto al ajuste hacia el largo plazo, El modelo de corrección de errores (MCE), señala que el coeficiente para las cinco ecuaciones resultó estadísticamente significativo y con el signo correcto; es decir, negativo; sin embargo, el valor de estos es muy bajo, lo que significa que el ajuste hacia el largo plazo, es sumamente lento. Esto, dado los profundos desequilibrios que afronta la economía venezolana.

Estos resultados son importantes puesto que puede permitir y, hasta validar el uso del gasto público como instrumento de política fiscal, lo que pudiera favorecer la reducción de la brecha entre el PIB potencial y el PIB. Mas allá de lo planteado, sería importante utilizar otras definiciones de gasto público y diferentes períodos de estudio, para validar o rechazar los resultados anteriores. En términos generales, esta investigación pudo aportar evidencia de la importancia del gasto público para el apuntalar el crecimiento, siempre y cuando ese gasto sea productivo. También, en una economía como la venezolana, con profundos desequilibrios, entre ellos los fiscales, los efectos positivos a largo plazo, de un aumento del gasto público se distorsionan, no logrando alcanzar los objetivos planteados.

\section{Notas}

${ }^{1}$ Si bien es cierto que Keynes no fue el primero en proponer esta relación; sin embargo, el economista británico ha sido quizás, el más 
destacado en sostener la perspectiva de la expansión del sector público para sustentar el crecimiento económico.

${ }^{2} \mathrm{Si}$ bien es cierto que Keynes no fue el primero en proponer esta relación; sin embargo, el economista británico ha sido quizás, el más destacado en sostener la perspectiva de la expansión del sector público para sustentar el crecimiento económico.

${ }^{3} \mathrm{La}$ posición keynesiana contempla que las políticas fiscales expansivas de gasto e inversión pública pueden ser aplicadas fuera de un entorno económico negativo, a través del impulso del crecimiento económico y la reducción del desempleo.

${ }^{4}$ La hipótesis de Warner tiene sentido si: a) Existe una relación de largo plazo entre las variables, es decir, dichas variables cointegran; b) que la causalidad vaya del PIB al gasto público; y, c) que el nexo entre al PIB y el gasto sea elástico.

${ }^{5}$ Hay que mencionar que, la diferencias en los resultados obtenidos, puede ser atribuido a las definiciones que se le da al gasto público.

${ }^{6}$ Esta es una variable propia del modelo de demanda agregada; además, una serie clásica en el análisis del gasto público.

7 Siempre que las variables incluidas en el modelo sean integradas de orden uno (I(1)), se podrá distinguir entre relaciones de corto y largo plazo e interpretar las de largo plazo, como relaciones de cointegración.

${ }^{8} 1989,2002$ y 2004 . Estas variables dummies resultaron estadísticamente significativas y recogen choques tanto estructurales como coyunturales.

${ }^{9}$ Este test es el más utilizado en la literatura econométrica. Presenta un mejor desempeño que otros test para casos de muestras pequeñas.

\section{Referencias bibliográficas}

Aschauer, D. A. (1989). ¿Is public expenditure productive? Journal of Monetary Economics, 23(2), 177-200. https://doi. org/10.1016/0304-3932(89)90047-0

Aparco, E., y Flores, A. (2019). La hipótesis
Keynesiana del gasto público frente a la Ley de Wagner: un análisis de cointegración y causalidad para Perú. Revista de Economía del Rosario, 22(1), 53-73. https://doi. org/10.12804/revistas.urosario.edu.co/ economia/a.7764

Baptista, A. (2011). Bases cuantitativas de la economía venezolana 1830 - 2008. Artesanogroup Editores.

Barro, R. J. (1990). Government spending in a simple model of endogeneous growth. Journal of Political Economy, 98(S5), 103-125. $\quad$ https://doi. org/10.1086/261726

Bird, R. (1971). Wagner's 'Law' of expanding state activity. Public Finance, 26(1), $1-26$.

Comín, F., Díaz, D., y Revuelta, J. (5-6 de febrero de 2009). La relación entre el crecimiento económico y el gasto público en Argentina, Brasil, España México durante el siglo XX. XVI Encuentro de Economía Pública, Granada, España.

Campo, J., y Mendoza, H. (2018). Gasto público y crecimiento económico: Un análisis regional para Colombia, 19842012. Lecturas de Economía, (88), 77-108. https://doi.org/10.17533/udea. le.n88a03

Diaz-Fuentes, D., y Revuelta, J. (2013). La relación a largo plazo entre crecimiento económico y gasto público en España (1850-2000). Investigaciones de Historia Económica, 9(1), 3242. $\quad$ https://doi.org/10.1016/j. ihe.2012.06.001

Dickey, D. A., y Fuller, W. A. (1979). Distribution of the estimators for autoregressive time series with a unit root. Journal of the American Statistical Association, 74(336), 427431. https://doi.org/10.2307/2286348

Dickey, D. A., y Fuller, W. A. (1981). 
Gasto público y crecimiento económico en Venezuela: Un análisis de cointegración y causalidad (1950 - 2017)

Likelihood ratio statistics for autoregressive time series with a unit root. Econometrica, 49(4), 1057-1072. https://doi.org/10.2307/1912517

Engle, R. F., y Granger, C. W. J. (1987). CoIntegration and error correction: Representation, estimation, and testing. Econometrica, 55(2), 251-276. https://doi.org/10.2307/1913236

Goffman, I. J. (1968). On the empirical testing of Wagner's Law: A technical note. Public Finance, 4(23), 359-364.

Granger, C. W. J. (1969). Investigating causal relations by econometric models and cross-spectral methods. Econometrica, 37(3), 424-438. https:// doi.org/10.2307/1912791

Granger, C. W. J. (1986). Developments in the study of cointegrated economic variables. OxfordBulletin of Economics and Statistics, 48(3), 213-228. https:// doi.org/10.1111/j.1468-0084.1986. mp48003002.x

Gupta, S.P. (1967). Public expenditure and economic growth: A time series. Analysis. Public Finance, 22, 423-461.

Henrekson, M. (1992). An economic analysis of Swedish government expenditure. Avebury.

Johansen, S. (1988). Statistical Analysis of Cointegration Vectors. Journal of Economic Dynamics and Control, 12(2-3), 231-254. $\quad$ https://doi. org/10.1016/0165-1889(88)90041-3

Johansen, S., y Juselius, K. (1990). Maximum likelihood estimation and inference on cointegration - with applications to the demand for money. Oxford Bulletin of Economics and Statistics, 52(2), 169-210. https:// doi.org/10.1111/j.1468-0084.1990. mp52002003.x

Kaur, K., y Afifa, U. (2017). Testing Wagner's Law in India: A cointegration and causality analysis. Communication in Statistics-Theory and Methods, 46(17), 8510-8520.

Keho, Y. (2016). Testing Wagner's Law in the presence of structural changes: New evidence from six African countries (1960-2013). International Journal of Economics and Financial Issues, 6(1), 1-6.

Keynes, J. M. (1926). The end of LaissezFaire. Hogarth Press.

Keynes, J. M. (1971). The collected writings of John Maynard Keynes. Macmillan.

Magazzino, C. (2012). Wagner versus Keynes: Public spending and national income in Italy. Journal of Policy Modeling, 34(6), 890-905. https://doi. org/10.1016/j.jpolmod.2012.05.012

Mann, A. J. (1980). Wagner's Law: An econometric test for Mexico, 19251976. National Tax Journal, 33(2), 189-201.

Molina, G., y Gantier, M. (2015). Ley de Wagner y Keynes: El caso de Bolivia. Documento de Trabajo, No. $11 / 15$. Instituto de Investigaciones Socio-Económicas (IISEC). http:// www.iisec.ucb.edu.bo/assets iisec/ publicacion/2015-10.pdf

Musgrave, R. A. (1969). Fiscal systems. Yale University Press.

Musgrave, R. A., y Peacock, A. (Eds.) (1958). Classics in the theory of public finance. Palgrave Macmillan. https:// doi.org/10.1007/978-1-349-23426-4

Peacock, A. T., y Wiseman, J. (1961). The growth of public expenditure in the United Kingdom. Princeton University Press.

Peña, C. (2018). Relación entre infraestructuracrecimiento económico en Venezuela, 1950-2008. Economía, XlII(46), 101124. 
Phillips, P. C. B., y Perron, P. (1988). Testing for a unit root in time series regression. Biometrika, 75(2), 335-346. https:// doi.org/10.2307/2336182

Salazar, C. A. (2020). Gasto público y crecimiento económico: Controversias teóricas y evidencia para México. Economía UNAM, 17(50), 53-71.

Singh, B., y Sahni, B. S. (1984). Causality between public expenditure and national income. Review of Economics and Statistics, 66(4), 630-644. https:// doi.org/10.2307/1935987

Vera, J. A., y Kristjanpoller, W. (2017).
Causalidad de Granger entre composición de las exportaciones, crecimiento económico y producción de energía eléctrica: evidencia empírica para Latinoamérica. Lecturas de Economía, (86), 25-62. https://doi. org/10.17533/udea.le.n86a02

Wagner, A. (1958). Three extracts on public finance. In R. A. Musgrave y A. T. Peacock (Eds.), Classics in the theory of public finance. International Economic Association Series (pp. 1-15). Palgrave Macmillan. https:// doi.org/10.1007/978-1-349-23426$\underline{4} 1$ 